\title{
Comparison of ovarian cancer markers in endometriosis favours HE4 over CA125
}

\author{
BRETT McKINNON ${ }^{1}$, MICHAEL D. MUELLER ${ }^{2}$, KONSTANTINOS NIRGIANAKIS $^{2}$ and NICK A. BERSINGER ${ }^{1,2}$ \\ Departments of ${ }^{1}$ Clinical Research and ${ }^{2}$ Obstetrics and Gynaecology, University of Berne, Berne CH-3010, Switzerland
}

Received September 26, 2014; Accepted June 2, 2015

DOI: $10.3892 / \mathrm{mmr} .2015 .4062$

\begin{abstract}
Endometriosis is a gynaecological condition with an associated chronic inflammatory response. The ectopic growth of 'lesions', consisting of endometrial cells outside the uterine cavity, stimulates an inflammatory response initiating the activation of macrophages, and resulting in increased cytokine and growth factor concentrations in the peritoneal fluid (PF). Endometriosis-associated inflammation is chronic and long lasting. In patients with endometriosis, the risk of developing ovarian cancer within 10 years, particularly of the endometrioid or clear cell subtype, is increased 2.5-4 times. Endometriosis creates a peritoneal environment that exposes the affected endometriotic and the normal ovarian surface epithelial cells to agents that have been suggested to be involved in the pathogenesis of cancer. Concentrations of several cytokines and growth factors were increased in the $\mathrm{PF}$ of patients with endometriosis. The ovarian cancer marker, CA125, was one such growth factor; however, this remains to be confirmed. Human epididymis protein 4 (HE4) was detected at high concentrations in patients with ovarian cancer and was identified as the best biomarker for the detection of ovarian cancer. The present study determined the levels of HE4 and CA125 in the peritoneal fluid of 258 patients with and 100 control individuals without endometriosis attending the Department of Obstetrics and Gynaecology, University of Berne (Berne, Switzerland) between 2007 and 2014. The cases were subdivided into groups without hormonal treatment $(n=107)$, or treated with combined oral contraceptives $(n=45)$, continuous gestagens $(n=56)$ or $\mathrm{GnRH}$ agonists $(n=50)$. Both of these markers were significantly increased in the non-treated endometriosis samples compared with the control group. Hormone treatment with either of the three agents mentioned resulted in the concentration of CA125 returning to the control levels and the concentration of HE4 decreasing to below the control levels. CA125, however not HE4, significantly differed
\end{abstract}

Correspondence to: Professor Nick A. Bersinger, University of Berne, 35 DKF Murtenstrasse, Berne CH-3010, Switzerland

E-mail: nick.bersinger@dkf.unibe.ch

Key words: endometriosis, ovarian cancer markers, CA125, HE4, peritoneal fluid between the proliferative and secretory cycle phases. Since HE4 is sensitive to hormonal treatment and robust towards menstrual cycle variation, HE4 is potentially superior to CA125 as an endometriosis marker and therefore has greater potential as a marker for the identification of women at risk of developing ovarian cancer.

\section{Introduction}

Endometriosis is an extremely prevalent gynaecological disorder defined by the presence and growth of endometrial tissue outside the uterine cavity. It occurs in $10-20 \%$ of females of reproductive age worldwide (1) and often results in severe pelvic pain (2) and reduced fecundity (3). It is a benign disease; however, it expresses malignant features, including angiogenesis, abnormal apoptotic patterns, unrestrained cellular proliferation and in rare cases, invasion of distant organs (4,5). Sampson's postulation (6) of retrograde menstruation of viable endometrial cells, which implant and grow at ectopic sites is still the most widely accepted theory for the histogenesis of endometriosis (7). The attachment of single cells to a substrate, their invasion, proliferation and vascularisation are properties that occur in carcinogenesis, and are similar or identical to the implantation of the embryo in the endometrium and to endometriosis.

An association between endometriosis and ovarian cancer has been previously demonstrated $(8,9)$. Due to a lack of effective early detection methods and minimal physical symptoms, epithelial ovarian cancer is commonly diagnosed at advanced stages, resulting in one of the highest mortality rates amongst all gynaecological malignancies (10). The use of oral contraceptives was demonstrated to be beneficial on the incidence of ovarian cancer $(11,12)$. A possible explanation for an increased rate of malignant transformation in the ovary is provided by the 'incessant ovulation' theory which, although suggested a number of years ago, is currently regaining importance. This theory suggests a negative impact of each ovulation (13) via the underlying inflammatory response associated with each occurrence (14). Pregnancy and the use of contraceptives suppressing ovulation would therefore reduce the risk of ovarian cancer $(15,16)$.

In the peritoneal fluid (PF) of patients with endometriosis, a number of cytokines, chemokines and growth factors have been demonstrated to be expressed at higher levels compared with females without the disease (17-21). The same has been suggested for the expression levels of CA125, a coelomic 
epithelial membrane glycoprotein antigen of unknown biological function, which is widely used as a serum marker for ovarian cancer and other types of tumour. Certain studies have suggested the importance of CA125 as a marker for endometriosis $(22,23)$ and other previous studies have confirmed this $(24,25)$. Additionally, CA125 has been demonstrated to be produced in vitro by endometrial tissue in explant cultures (26). CA125 is an established serum marker for epithelial ovarian cancer $(27,28)$. Serum CA125 has also been demonstrated to be increased in non-malignant disorders (29). The correlation between serum and PF CA125 has been previously demonstrated (25). Human epididymis protein 4 (HE4), a small glycoprotein and protease inhibitor first identified in epididymal epithelium, has been identified as an ovarian cancer marker, particularly for the serous and endometrioid type (30), and is superior to CA125 (31) either on its own or in combination with CA125 as defined by a higher detection rate (32-34). The increased specificity of HE4 over CA125 was previously confirmed in a large patient cohort with benign diseases (35).

The current available information regarding HE4 in the context of endometriosis in the literature is scarce and pertains exclusively to its concentration in the circulation. Additionally, to the best of our knowledge, no information exists with regards to HE4 in the PF of patients with endometriosis. The present study aimed to investigate the two ovarian cancer markers, CA125 and HE4, in the PF of patients with endometriosis, with or without treatment with oral contraceptives (combined or gestagen-only) or with GnRH agonists, and control individuals.

\section{Materials and methods}

Patient information. A total of 358 women were involved in the present study. They underwent laparoscopic surgery in the Department of Obstetrics and Gynaecology, University of Berne (Berne, Switzerland) between July 2007 and July 2014 for reasons of chronic abdominal or menstrual pain, or as a result of unexplained infertility. Information regarding the presence and staging, or absence of endometriosis, hormonal treatment administration, and/or menstrual cycle stage was obtained together with the biological samples, or retrieved post hoc from the medical records. All enrolled women provided informed, written consent. The study was approved by the ethics committee of the Canton of Berne (approval no. KEK14903).

Patient exclusion criteria. PF was quantitatively collected from the Pouch of Douglas and clarified by centrifugation at $800 \mathrm{x} \mathrm{g}$ for $10 \mathrm{~min}$. The supernatant was stored at $-80^{\circ} \mathrm{C}$ in aliquots after recording the total volume. The total protein content was determined using a micro-bicinchoninic assay (Quanti-Pro ${ }^{\circledR}$ BCA, Sigma-Aldrich, St. Louis, MO, USA) to ascertain absence of dilution with abdominal flushing medium under the procedure. The PF samples with a total protein content of $<10 \mathrm{mg} / \mathrm{ml}$ were excluded from the present study. Other exclusion criteria were as follows: The presence of haemolysis in the PF, the diagnosis of malignancies or the use of hormonal therapy in the three months prior to the procedure.
A total of 258 patients with endometriosis and 100 control individuals without endometriosis were included. The group of cases included patients who did not receive any hormonal treatment in the 3 months prior to laparoscopy $(n=107)$. Of these patients, 67 were in the proliferative and 28 were in the secretory phase of the menstrual cycle (cycle stage was peri-ovulatory or unknown in 12 cases). The remaining cases were divided into three groups: Treatment with combined oral contraceptives $(n=45)$, continuous progesterone $(n=56)$ or GnRH agonists ( $\mathrm{n}=50)$, all for at least 3 months leading up to the day of surgery. The control group (without endometriosis, $\mathrm{n}=100$ ) consisted of 67 women in the proliferative menstrual cycle stage and 33 in the secretory menstrual cycle stage. The demographic information and PF characteristics are shown in Table I.

ELISA. CA125 and HE4 were quantified manually in the PF samples using commercially available microplate ELISA kits. For CA125, the TM-CA125 ELISA kit (cat. no. EIA-5072; DRG Instruments, Marburg, Germany) was used, according to the manufacturer's instructions. Incubation temperature was maintained at $28^{\circ} \mathrm{C}$ without agitation. The functional sensitivity was $0.25 \mathrm{U} / \mathrm{ml}$ and the intra- and inter-assay coefficients of variance at $30 \mathrm{U} / \mathrm{ml}$ were 5.8 and $10.6 \%$, respectively. The PF samples were diluted 1:21 with phosphate-buffered saline, containing $0.1 \%(\mathrm{w} / \mathrm{v})$ bovine serum albumin (Sigma-Aldrich, Buchs, Switzerland). For HE4, the EIA kit 404-10 (Fujirebio $\mathrm{AB}$, Göteborg, Sweden) was used, according to the manufacturer's instructions. Incubations were performed at $28^{\circ} \mathrm{C}$ with an agitation speed of $300 \mathrm{rpm}$. The detection limit was $15 \mathrm{pM}$ (functional sensitivity, $<2.5 \mathrm{pM}$ ), and the coefficient of variance (intra-assay) was $2.4 \%$. The PF samples were diluted 1:26 with the zero calibrator of the assay kit.

Statistical analysis. The concentrations of CA125 and HE4 were compared non-parametrically between the different groups using Mann-Whitney U test. Correlation analyses were performed using Spearman's rank correlation, following log transformation of the X (HE4) and Y (CA125) axes, using Graph-Pad Prism ${ }^{\circledR}$ v.5.04 software (GraphPad Software, Inc., La Jolla, CA, USA). $\mathrm{P}<0.05$ was considered to indicate a statistically significant difference.

\section{Results}

Levels of CA125 and HE4 vary in different patient samples. The levels of CA125 and HE4 were below the above mentioned detection limits in 2/358 and 1/358 PF samples, respectively. In the non-parametrical statistical analysis these values were included as $10 \mathrm{U} / \mathrm{ml}$ for CA125 and $100 \mathrm{pmol} / 1$ for HE4. All results for CA125 and HE4 in the different groups and sub-groups are shown in Table II. Marked patient-to-patient variations for the two markers were detected over two (CA125 in controls only, <2) to three orders of magnitude. This is shown for CA125 and HE4 in Fig. 1A and B, respectively.

Levels of CA125 and HE4 are affected by hormone treatment. The concentrations of the markers were demonstrated to be significantly increased in the group of non-treated endometriosis cases compared with the controls without 
Table I. Demographic data and PF characteristics.

\begin{tabular}{|c|c|c|c|c|c|}
\hline Characteristic & $\mathrm{N}$ & Age (years) & BMI $\left(\mathrm{kg} / \mathrm{m}^{2}\right)$ & PF volume (ml) & PF protein $(\mathrm{mg} / \mathrm{ml})$ \\
\hline No endometriosis & 100 & $34.3 \pm 8.0$ & $24.4 \pm 4.0$ & $9.7 \pm 8.0$ & $34.47 \pm 10.63$ \\
\hline Proliferative phase & 67 & $33.6 \pm 8.0$ & $24.3 \pm 4.5$ & $8.5 \pm 8.1$ & $32.96 \pm 9.87$ \\
\hline Secretory phase & 33 & $35.7 \pm 7.9$ & $24.7 \pm 3.0$ & $12.2 \pm 7.3$ & $37.57 \pm 11.59$ \\
\hline Endometriosis & 258 & & & & \\
\hline Not treated & 107 & $34.5 \pm 5.8$ & $23.7 \pm 4.0$ & $10.1 \pm 8.1$ & $34.18 \pm 10.29$ \\
\hline Proliferative phase & 67 & $33.6 \pm 5.7$ & $23.8 \pm 3.9$ & $9.5 \pm 8.0$ & $31.60 \pm 10.39$ \\
\hline Secretory phase & 28 & $35.2 \pm 5.2$ & $22.9 \pm 3.3$ & $12.6 \pm 8.7$ & $38.62 \pm 7.03$ \\
\hline Peri-ovulatory or unknown & 12 & & & & \\
\hline OC treated & 45 & $28.7 \pm 5.1$ & $22.3 \pm 4.4$ & $7.2 \pm 5.9$ & $33.19 \pm 8.00$ \\
\hline Gestagen only treated & 56 & $31.5 \pm 5.9$ & $22.6 \pm 3.3$ & $6.9 \pm 6.5$ & $38.46 \pm 8.25$ \\
\hline GnRHa treated & 50 & $31.6 \pm 5.7$ & $22.2 \pm 3.6$ & $8.1 \pm 7.6$ & $36.78 \pm 7.67$ \\
\hline
\end{tabular}

Data are expressed as the mean \pm standard deviation. PF, peritoneal fluid; BMI, body mass index; OC, oral contraceptives; GnRHa, GnRH agonist.

Table II. Ovarian cancer marker concentrations in peritoneal fluid.

\begin{tabular}{|c|c|c|c|c|c|c|}
\hline Characteristic & $\mathrm{N}$ & $\begin{array}{l}\text { CA-125 }(\mathrm{U} / \mathrm{ml}) \\
\text { median (range) }\end{array}$ & P-value & $\begin{array}{l}\mathrm{HE}-4(\mathrm{pmol} / \mathrm{l}) \\
\text { median (range) }\end{array}$ & P-value & $\begin{array}{l}\text { CA-125xHE-4 } \\
\text { P-value }\end{array}$ \\
\hline No endometriosis & 100 & 806 (131-8798) & $0.0185^{\mathrm{a}}$ & $4731(<100-27543)$ & $0.0105^{\mathrm{a}}$ & $0.0061^{\mathrm{a}}$ \\
\hline Proliferative phase & 67 & $1064(139-5625)$ & & $5371(<100-27543)$ & & \\
\hline Secretory phase & 33 & $566(131-8798)$ & $0.0020^{\mathrm{b}}$ & $2731(103-24138)$ & $0.1826^{\mathrm{b}}$ & $0.0394^{\mathrm{b}}$ \\
\hline Endometriosis & 258 & & & & & \\
\hline Not treated & 107 & $1114(<10-12229)$ & & $6667(162-68506)$ & & \\
\hline Proliferative phase & 68 & $1416(72-12229)$ & & $6707(162-68506)$ & & \\
\hline Secretory phase & 30 & $818(<10-8853)$ & $0.0175^{\mathrm{b}}$ & $6641(567-21918)$ & $0.6555^{\mathrm{b}}$ & $0.3969^{b}$ \\
\hline OC treated & 45 & $762(<10-2709)$ & $0.0178^{c}$ & $1652(138-13274)$ & $<0.0001^{\mathrm{c}}$ & $<0.0001^{\mathrm{c}}$ \\
\hline Gestagen only treated & 56 & $865(128-5602)$ & $0.0233^{\mathrm{c}}$ & $1306(112-22782)$ & $<0.0001^{\mathrm{c}}$ & $<0.0001^{\mathrm{c}}$ \\
\hline GnRHa treated & 52 & $926(85-9734)$ & $0.2419^{c}$ & $1546(<100-34123)$ & $<0.0001^{\mathrm{c}}$ & $<0.0001^{\mathrm{c}}$ \\
\hline
\end{tabular}

Data are expressed as the median and range. P-values were calculated by Mann-Whitney U test. ${ }^{a}$ Controls (no endometriosis, N=100), vs. non-treated endometriosis $(\mathrm{N}=107)$; ${ }^{\mathrm{b}} \mathrm{Sec}$ etory, vs. proliferative cycle phase in the same group; ${ }^{\mathrm{c} C a l c u l a t e d}$, vs. non-treated endometriosis. OC, oral contraceptives; GnRHa, GnRH agonist.

any sign of the disease. The P-values were calculated for CA125 and HE4, and were revealed to be 0.0185 and 0.0105 , respectively (Mann-Whitney $\mathrm{U}$ test). Within the endometriosis cases, exposure to combined oral contraceptives and continuous progesterone resulted in the PF levels of CA125 returning to that of the control group $(\mathrm{P}=0.02)$. No effect on CA125 was observed following treatment with GnRH agonists. The levels of HE4 were markedly decreased in all three hormone-treated groups compared with the non-treated controls $(\mathrm{P}<0.0001)$, and were even lower compared with the levels observed in the control group (Table II). CA125 significantly differed in concentration between the proliferative and secretory cycle phases in the controls and non-hormone-treated cases of endometriosis; however, this was not the case for HE4 (Fig. 1).
Effect of assessing both markers. The product of both markers (HE4 x CA125) was also compared between the different groups. When the non-treated endometriosis cases were compared with the control group, the increase in this product was more pronounced $(\mathrm{P}=0.0061)$ compared with the two markers individually (Table II). When comparing proliferative and secretory phases the results for the product came to lie between those for the individual markers and the difference was significant in the control but not in the untreated endometriosis group. Conversely, the hormone treated groups demonstrated markedly decreased values for the product ( $\mathrm{P}<0.0001$ for all three hormones).

The concentrations of the two markers were revealed to be correlated with each other in all five groups and subgroups. Spearman P-values were between 0.0101 (gestagen treated 

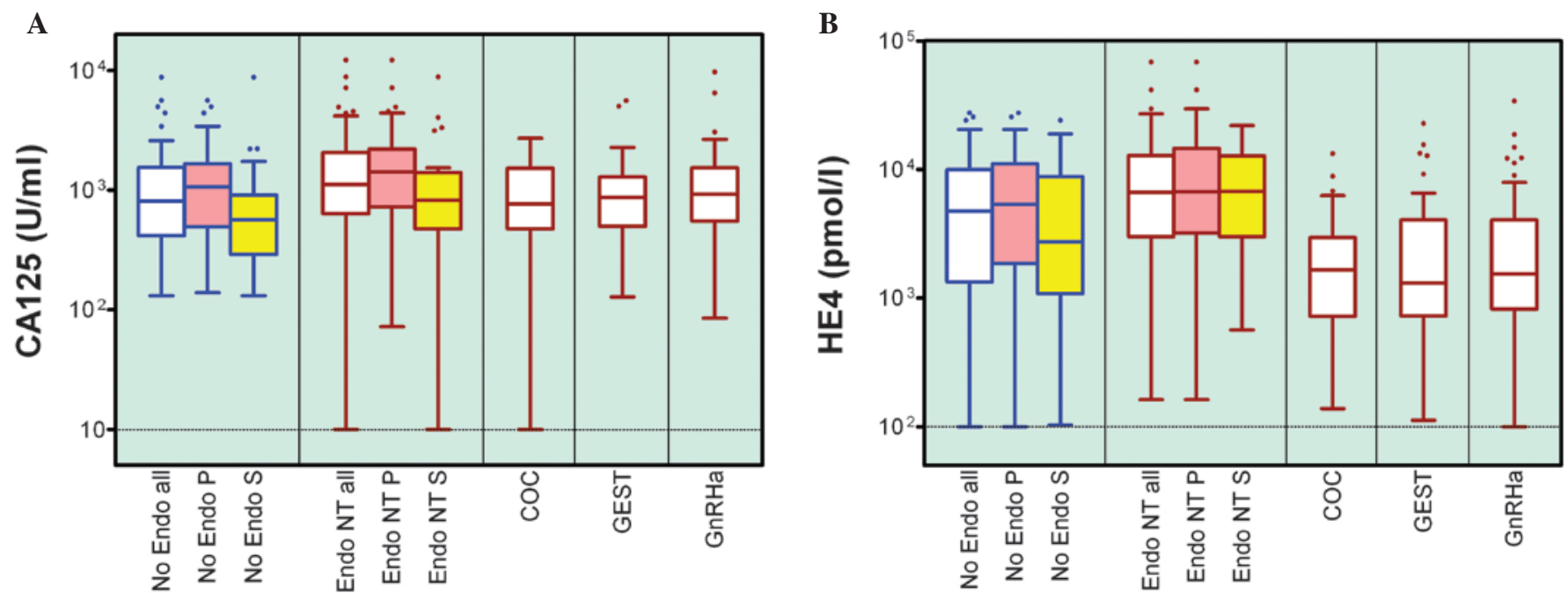

Figure 1. Tukey box and whisker plot of (A) CA125 and (B) HE4 concentrations in the PF of No Endo women, patients with endometriosis without hormonal treatment, under treatment with COC, GEST or GnRHa for at least 3 months prior to PF collection. Number of women per group and statistical significance are stated in Table II. P, proliferative phase of the menstrual cycle; S, secretory phase; PF, peritoneal fluid; No Endo, endometriosis-free; NT, non-treated; COC, combined oral contraceptives; GEST, gestagen-only; GnRHa, GnRH agonist.

\section{Correlation: All women}

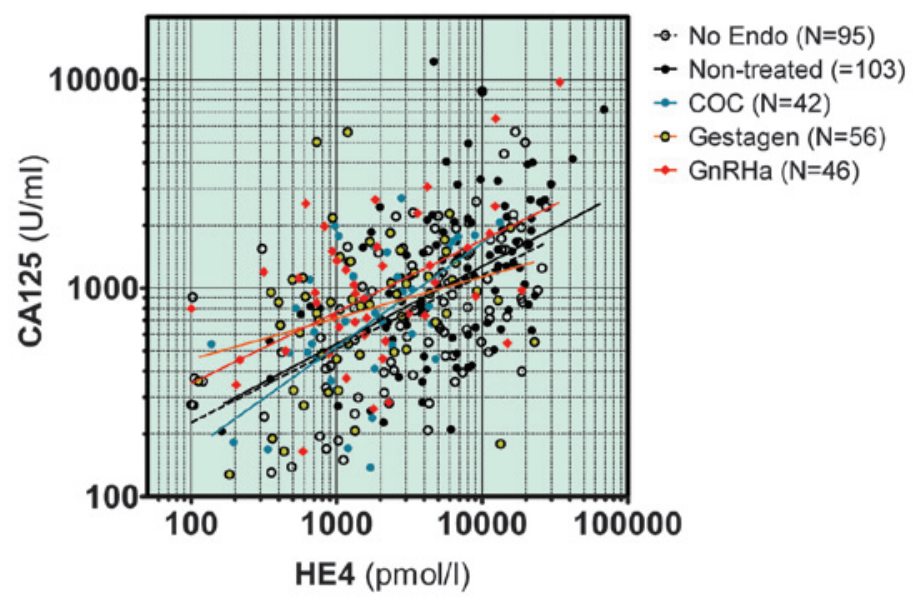

Figure 2. Correlation between the concentrations of CA125 and HE4 in the peritoneal fluid of 342 women. The regression lines were calculated linearly following double logarithmic transformation. All correlations, determined by Spearman Rank analysis, were statistically significant (P<0.01). No Endo, endometriosis-free; COC, combined oral contraceptives; GnRHa, GnRH agonist.

group; $r=0.3411$ ) and $<0.0001$ (controls; $r=0.5816$ ). The Spearman r-values were not identified to be significantly different between the five populations. These findings are shown in Fig. 2, following logarithmic transformation.

\section{Discussion}

The present study findings not only confirm increased PF concentrations of the ovarian cancer marker CA125 in patients with endometriosis $(24,25)$, but also demonstrate for the first time, to the best of our knowledge, similarly increased levels of the novel marker HE4 in this compartment. The two markers are shown to be closely correlated ( $\mathrm{P}<0.01$; Fig. 2 ), which is consistent with the results of a previous study that measured the concentration of the two markers in the serum of patients with ovarian cancer (36). Therefore, it may not be necessary to determine each of these markers, however, this will depend on the question under investigation. For the monitoring of an existing or suspected ovarian malignant pathology, it was demonstrated that the combination of the two markers provided an improved result compared with a single marker $(32,34)$. The findings of the present study confirm that the multiplication product (CA125 x HE4, in arbitrary units) provides an improved ability to discriminate between cases and controls ( $\mathrm{P}=0.0061$; Table II). No information exists in the literature regarding the levels of HE4 in the PF of patients with endometriosis; however, a small number of previous studies have identified HE4 in the serum. A previous case report associated increasing levels of CA125, and low and stable serum levels of HE4 with the disease (37) indirectly, suggesting unchanged HE4 levels in endometriosis. A previous study observed no differences in the serum levels of HE4 between patients with endometriosis and controls, 
or between the proliferative and secretory menstrual cycle phases (38). The same result was obtained following different hormone treatments, however, in these groups, particularly for GnRHa, the number of patients assessed was significantly smaller compared with that of the present study. All these absences of variations were advocated as an advantage of the HE4 test compared with the CA125 in patients with suspected ovarian cancer. A previous study investigated a large patient cohort (>1,000 patients) with benign gynaecological diseases, including endometriosis, and revealed an increased specificity of HE4 over CA125 as a result of less frequently increased levels in these females, particularly in the pre-menopausal group (35). The present findings in the PF are parallel with a previous study in the serum (38) regarding the cycle phases and the two markers, however, not for HE4 regarding the presence of endometriosis. Another previous study compared the serum levels of these markers between ovarian cancer and different endometriotic pathologies, and demonstrated that measuring both markers in the same sample made it possible to distinguish between the two pathologies, since HE4 but not CA125, was shown to be increased in endometriotic pathology (36). The mentioned study has the advantage of including cancerous and endometriosis groups, however, hormone exposure or cycle variations were not investigated. Further studies observing serum and PF from the same females are required to determine whether the differences between the present results and the few reports in the literature regarding HE4 increases in endometriosis are a consequence of the different compartments assessed. If so, the serum test for HE4 will be robust towards (independent from) menstrual cycle parameters or endometriotic pathologies; however, care may be required to ensure that no hormonal medication was used, since HE4 reacts even more significantly to these treatments compared with CA125.

Notably, a significant difference in the CA125 levels was demonstrated between the proliferative and secretory cycle phases in the controls and cases (which have not been exposed to hormonal treatment in the 3 months prior to PF collection). This is not in agreement with a previous report (24) in which the sample numbers were lower (11-22) and the assay was based on a different, older antibody, which may be less specific. Care is required and the cycle phase must be taken into account. The present study hypothesised that HE4 (which in this study, in contrast to CA125, failed to distinguish between the cycle phases), may be a more 'robust' marker individually when the cycle phase is unknown or the population is distributed over the entire menstrual cycle. However, CA125 and HE4 may exhibit better predictive ability alone when an exclusively proliferative or an exclusively secretory phase population is investigated, respectively (Table II). However, this requires further confirmation using larger groups of samples and patients.

Treatment of endometriosis may reduce the risk of developing ovarian cancer. Current treatments include the administration of contraceptives, combined-sequential or continuous, which inhibit ovulation and therefore, an additional inflammatory episode on the surface of the ovary and possibly in surrounding tissues. The use of contraceptives has also been demonstrated to reduce ovarian cancer risk independently of the presence of endometriosis (11). The present study demonstrates reduced levels of CA125 and significantly reduced levels of HE4. This suggests the usefulness of contraceptives, including gestagens, when administered alone in the treatment of endometriosis and potentially in the context of ovarian cancer, since these two proteins are associated with this pathology. In the present study the patients with endometriosis were not sub-grouped as a function of lesion location, however, our previous study observed that women were less likely to develop ovarian lesions compared with lesions in other locations (peritoneal or recto-vaginal) following the use of oral contraceptives (39). This is particularly notable in the context of the ovarian cancer risk discussed in the present study. Gonadotropin releasing hormone agonists (GnRHa) induce a hypo-estrogenic state and therefore can be used in the treatment of endometriosis as long as the patient does not wish to become pregnant; this is also the case with other hormonal medications. The increased incidence of ovarian cancer in patients with endometriosis provides a reason to identify endometriosis as soon as it occurs. It is difficult to suggest whether the sub-population with an increased risk may indeed be specifically characterised by increased levels of the ovarian cancer markers, CA125 and possibly HE4, in the phase of pre-malignancy. The present study, nevertheless, suggests that the treatment of endometriosis is required as a result of its potential to reduce the risk of ovarian cancer, as well as to reduce symptoms such as pain.

The present results confirm those of a previous study (40) demonstrating that GnRHa, administered for the reduction of pain (41), resulted in a significant reduction in peritoneal pro-inflammatory cytokine and growth factor levels, therefore mediating a regression in the inflammatory activity in the peritoneal environment in patients with endometriosis (42). The present study shows that several pro-inflammatory cytokines and growth markers exhibit reduced PF concentrations following GnRHa treatment in patients with endometriosis (42). It also reveals that CA125 levels are not reduced following such treatment while those of HE4 are $(\mathrm{P}<0.0001)$. $\mathrm{HE} 4$, therefore, may be an ideal marker, superior to CA125, for the success of medical treatment of endometriosis, since it responds similarly to the different categories of hormones and, as mentioned previously, does not depend on the menstrual cycle phase in the group of non-treated patients.

A drawback of the present study is the absence of data regarding the occurrence of ovarian cancer in the study population. It is extremely difficult to gather samples from patients with ovarian cancer, together with the complete information regarding the presence or absence of past endometriosis. Additionally, such a project would likely further benefit from the analysis of serum samples obtained during the endometriosis and the cancer phases. This was not performed in the present study where an increased specificity by the restriction to the peritoneal compartment was targeted.

In conclusion, the present study shows that the treatment of patients with endometriosis with sex steroids or GnRHa reduced the exposure of ovarian epithelial cells to an inflammatory environment. Whether this may later have a beneficial, negative effect on the risk of developing ovarian cancer remains to be elucidated. The response of the peritoneal environment to such treatment may be monitored by ovarian cancer markers, including CA125 and HE4, with the latter being slightly superior to the former since it does not react to 
menstrual cycle variations and the reductions in PF concentrations are more pronounced. Variance of the results within one group, however, is not better (smaller) for HE4 than for CA125. Long-term studies, including observation of serum levels, are required to gain further insight.

\section{Acknowledgements}

The authors would like to thank Dr Magnus Lövenklev (Fujirebio Diagnostics, Gothenburg, Sweden) for providing the assay kits for HE4 free of charge, Ms. Barbara Haldemann and her team for the collection of the PF samples and to Ms. Anne Vaucher for technical assistance in the laboratory. This study was funded, in part, by the Swiss National Science Foundation (grant no. 320030_140774).

\section{References}

1. Eskenazi B and Warner ML: Epidemiology of endometriosis. Obstet Gynecol Clin North Am 24: 235-258, 1997.

2. Evans S, Moalem-Taylor G and Tracey DJ: Pain and endometriosis. Pain 132 (Suppl 1): S22-S25, 2007.

3. D'Hooghe TM, Debrock S, Hill JA and Meuleman C: Endometriosis and subfertility: Is the relationship resolved. Semin Reprod Med 21: 243-254, 2003.

4. Chung SY, Kim SJ, Kim TH, et al: Computed tomography findings of pathologically confirmed pulmonary parenchymal endometriosis. J Comput Assist Tomogr 29: 815-818, 2005.

5. Fraser IS: Recognising, understanding and managing endometriosis. J Reprod Sci 1: 56-64, 2008.

6. Sampson J: Peritoneal endometriosis due to the menstrual dissemination of endometrial tissue into the peritoneal cavity. Am J Obstet Gynecol 14: 422-469, 1927.

7. Halme J, Hammond M, Hulka J, Raj S and Talbert L: Retrograde menstruation in healthy women and in patients with endometriosis. Obstet Gynecol 64: 151-154, 1984.

8. Brinton LA, Gridley G, Persson I, Baron J and Bergqvist A: Cancer risk after a hospital discharge diagnosis of endometriosis. Am J Obstet Gynecol 176: 572-579, 1997.

9. Swiersz L: Role of endometriosis in cancer and tumour development. Ann NY Acad Sci 955: 281-292, 2002.

10. Martin DC: Cancer and endometriosis: Do we need to be concerned? Semin Reprod Endocrinol 15: 319-324, 1997.

11. Gwinn ML, Lee NC, Rhodes PH, Layde PM and Rubin GL: Pregnancy, breast feeding, and oral contraceptives and the risk of epithelial ovarian cancer. J Clin Epidemiol 43: 559-568, 1991.

12. Fraser IS and Kovacs GT: The efficacy of non-contraceptive uses for hormonal contraceptives. Med J Aust 178: 621-623, 2003.

13. Fathalla MF: Incessant ovulation - a factor in ovarian neoplasia? Lancet 2: 163, 1971.

14. Ness RB, Cramer DW, Goodman MT, et al: Infertility, fertility drugs, and ovarian cancer: A pooled analysis of case-control studies. Am J Epidemiol 155: 217-224, 2002.

15. Booth M, Beral V and Smith P: Risk factors for ovarian cancer: A case-control study. Br J Cancer 60: 592-598, 1989.

16. Risch HA, Marrett LD and Howe, GR: Parity, contraception, infertility, and the risk of epithelial ovarian cancer. Am J Epidemiol 140: 585-597, 1994.

17. Ryan IP, Tseng JF, Schriock ED, Khorram O, Landers DV and Taylor RN: Interleukin-8 concentrations are elevated in peritoneal fluid of women with endometriosis. Fertil Steril 63: 929-932, 1995.

18. Lebovic DI, Mueller MD and Taylor RN: Immunobiology of endometriosis. Fertil Steril 75: 1-10, 2001

19. Bersinger NA, von Roten S, Wunder DM, Raio L, Dreher E and Mueller MD: PAPP-A and osteoprotegerin, together with interleukin-8 and RANTES, are elevated in the peritoneal fluid of women with endometriosis. Am J Obstet Gynecol 195: 103-108, 2006.

20. Siristatidis C, Nissotakis C, Chrelias C, Iacovidou H and Salamalekis E: Immunological factors and their role in the genesis and the development of endometriosis. J Obstet Gynaecol Res 32: 162-170, 2006.
21. Bersinger NA, Dechaud H, Mckinnon B and Mueller MD: Analysis of cytokines in the peritoneal fluid of endometriosis patients as a function of the menstrual cycle stage using the Bio-Plex platform. Arch Physiol Biochem 118: 210-218, 2012

22. Moen MH, Hagen B and Onsrud M: CA-125 in peritoneal fluid from patients with endometriosis. Hum Reprod 6, 1400-1403, 1991.

23. Koninckx PR, Riitinen L, Seppala M and Cornillie FJ: CA-125 and placental protein 14 concentrations in plasma and peritoneal fluid of women with deeply infiltrating pelvic endometriosis. Fertil Steril 57: 523-530, 1992.

24. Matalliotakis IM, Goumenou AG, Mulayim N, Karkavitsas N and Koumantakis EE: High concentrations of the CA-125, CA 19-9 and CA 15-3 in the peritoneal fluid between patients with and without endometriosis. Arch Gynecol Obstet 271: 40-45, 2005.

25. Amaral VF, Ferriani RA, Sá MF, Nogueira AA, Rosa e Silva JC, Rosa e Silva AC and Moura MD: Positive correlation between serum and peritoneal fluid CA-125 levels in women with pelvic endometriosis. Sao Paulo Med J 124: 223-227, 2006.

26. Bersinger NA, Sinosich MJ, Baber R, Torode H and Saunders DM: Development of an endometrial explant model for the investigation of uterine readiness for implantation in the human. In: Implantation in Mammals, Serono Symposia 91: 301-308, 1993.

27. Duffy MJ: Role of tumour markers in patients with solid cancers. A critical review. Eur J Intern Med 18: 175-184, 2007.

28. Anderson MR, Goff BA, Lowe KA, et al: Combining a symptoms index with CA125 to improve detection of ovarian cancer. Cancer 113: 484-489, 2008.

29. Ataseven H, Oztürk ZA, Arhan M, et al: Cancer antigen 125 levels in inflammatory bowel diseases. J Clin Lab Anal 23: 244-248, 2009.

30. Drapkin R, Von Horsten HH, Lin Y, et al: Human epididymis protein 4 (HE4) is a secreted glycoprotein that is overexpressed by serous and endometrioid ovarian carcinomas. Cancer Res 65 : 2162-2169, 2005

31. Hellstrom I, Raycraft J, Hayden M, et al: The HE4 (WFDC2) protein is a biomarker for ovarian carcinoma. Cancer Res 63 , 3695-3700, 2003.

32. Moore RG, Brown AK, Miller MC, et al: The use of multiple novel tumor biomarkers for the detection of ovarian carcinoma in patients with a pelvic mass. Gynecol Oncol 108: 402-408, 2008.

33. Chang X, Ye X, Dong L, et al: Human epididymis protein 4 (HE4) as a serum tumor biomarker in patients with ovarian carcinoma. Int J Gynecol Cancer 21: 852-858, 2011.

34. Escudero JM, Auge JM, Filella X, Torne A, Pahisa J and Molina R: Comparison of serum human epididymis protein 4 with cancer antigen 125 as a tumor marker in patients with malignant and nonmalignant diseases. Clin Chem 57: 1534-1544, 2011.

35. Moore RG, Miller MC, Steinhoff MM, et al: Serum HE4 levels are less frequently elevated than CA125 in women with benign gynecologic disorders. Am J Obstet Gynecol 206: 351.e1-e8, 2012.

36. Huhtinen K, Suvitie P, Hiissa J, et al: Serum HE4 concentration differentiates malignant ovarian tumours from ovarian endometriotic cysts. Br J Cancer 100: 1315-1319, 2009.

37. Anastasi E, Granato T, Coppa A, et al: HE4 in the differential diagnosis of a pelvic mass: a case report. Int J Mol Sci 12: 627-632, 2011.

38. Hallamaa M, Suvitie P, Huhtinen K, Matomäki J, Poutanen M and Perheentupa A: Serum HE4 concentration is not dependent on menstrual cycle or hormonal treatment among endometriosis patients and healthy premenopausal women. Gynecol Oncol 125: 667-672, 2012

39. McKinnon BD, Bertschi D, Wanner J, Bersinger NA and Mueller MD: Hormonal contraceptive use and the prevalence of endometriotic lesions at different regions within the peritoneal cavity. Biomed Res Int 2014: 590950, 2014.

40. Kupker W, Schultze-Mosgau A and Diedrich K: Paracrine changes in the peritoneal environment of women with endometriosis. Hum Reprod Update 4: 719-723, 1998.

41. Brown J, Pan A and Hart RJ: Gonadotrophin-releasing hormone analogues for pain associated with endometriosis. Cochrane Database Syst Rev CD008475, 2010.

42. Nirgianakis K, Bersinger NA, McKinnon B, Kostov P, Imboden S and Mueller MD: Regression of the inflammatory microenvironment of the peritoneal cavity in women with endometriosis by GnRHa treatment. Eur J Obstet Gynecol Reprod Biol 170: $550-554,2013$ 